\title{
Glosse
}

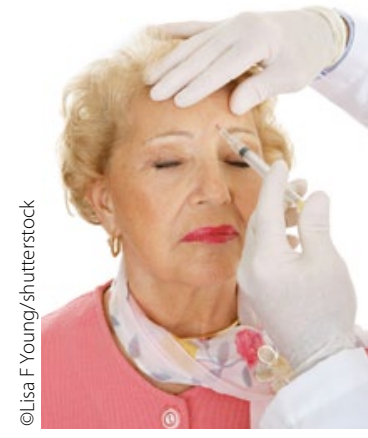

Wirksam gegen Frust und Falten.

\section{Botox für die Seele}

- Als eifriger MMW-Leser werden Sie sich vielleicht erinnern. Vor einigen Wochen wurde in diesem Journal über eine Studie berichtet, deren Ergebnisse zwischenzeitlich auch die Boulevardpresse erreicht haben. Auf einen kurzen Nenner gebracht konnte gezeigt werden, dass die Injektion von Botulinumtoxin in die Stirnhaut eine wirksame antidepressive Therapie darstellt. Man könnte sagen: Botox glättet nicht nur die Falten im Gesicht, sondern auch im Gehirn.

Bei der Konnotation von Gehirn und Botox hatte ich bisher eher den Verdacht, dass bei der einen oder anderen mit Botox aufgeschönten Dame vielleicht die Nadel doch etwas zu tief geraten sein könnte. Anders als mit einer hirnorganischen Störung lässt sich nämlich der Sinngehalt mancher Interviewaussagen kaum erklären. Dass aber das Wegspritzen der Stirnfurchen ohne direkte Berührung des Gehirns die Stimmung aufhellt, ist schon ein Knüller! Somit dürfte das Krankheitsbild der Depression jetzt von einer psychiatrischen zu einer dermatologischen Herausforderung mutieren. Besonders vorteilhaft ist, dass mit dieser neuen Methode Fühlen und Aussehen in Einklang gebracht werden: Der Patient ist nicht nur heiter gestimmt, sondern sieht auch so aus. Das Gesicht wird zum Spiegel der Seele. Und dies lässt sich mit Antidepressiva ja wohl kaum erreichen.

Die topische Behandlung mit Botox eröffnet aber auch neue Perspektiven für andere Erkrankungen. Vielleicht kann die Spritze in den Popo oder die Bauchdecke ja auch bei einer hartnäckigen Obstipation helfen.

Dr. med. Peter Stiefelhagen .

\section{GEHEIMREZEPT FÜR AKNEPATIENTEN?}

\section{Mehr Fisch, weniger Milch!}

- Abspecken, wenig Milch trinken, mehr Fisch essen - so könnte einer italienischen Studie zufolge das Rezept für eine bessere Haut bei Aknepatienten lauten. In der FallKontroll-Studie wurden 205 Patienten zwischen 10 und 24 Jahren mit "mittelschwerer" oder "schwerer Akne" befragt. Die Kontrollgruppe bildeten 358 Patienten mit leichter oder ohne Akne. Die Interviewer fragten u. a. nach Essgewohnheiten, Menstruation, Kontrazeptiva, Alkohol- und Zigarettenkonsum und berechneten den BMI.

Am höchsten war das Risiko für eine schwerere Akne, wenn Angehörige ersten Grades ebenfalls an der Erkrankung litten (Odds Ratio, OR 3,41). Aber auch bei der Ernährung zeigten sich Auffälligkeiten: So war das Aknerisiko höher, wenn die Jugendlichen mehr als drei Portionen Milch, v. a. Magermilch, pro Woche zu sich nahmen $(O R \quad 1,78)$. Verglichen mit einem BMI unter $18,5 \mathrm{~kg} / \mathrm{m}^{2}$ stieg die OR auf 1,9, wenn der BMI zwischen 18,5 und $23 \mathrm{~kg} / \mathrm{m}^{2}$ lag und auf 1,95, wenn er über $23 \mathrm{~kg} / \mathrm{m}^{2}$ erreichte. Ein niedriger BMI dagegen ging mit einem geringeren Aknerisiko einher. Auch wer regelmäßig Fisch auf dem Teller hatte, erlebte eine gewisse Schutzwirkung auf seine Haut (OR 0,68).

Keinen Einfluss hatten das Rauchverhalten oder verschiedene Belange der Menstruation. Auch ergaben sich bei anderen Lebensmitteln keine signifikanten Zusammenhänge. Selbst die viel diskutierten Süßigkeiten, Kuchen und Schokolade ließen die Pickel nicht stärker sprießen. ST •

Di Landro. JAAD 2012. Online first 3 March 2012 